\title{
Tissue Object Patterns for Segmentation in Histopathological Images
}

\author{
Cigdem Gunduz-Demir \\ Department of Computer Engineering \\ Bilkent University \\ Ankara, Turkey \\ gunduz@cs.bilkent.edu.tr
}

\begin{abstract}
In the current practice of medicine, histopathological examination is the gold standard for routine clinical diagnosis and grading of cancer. However, as this examination involves the visual analysis of biopsies, it is subject to a considerable amount of observer variability. In order to decrease the variability, it has been proposed to develop systems that mathematically model the histopathological tissue images and automate the analysis. Segmentation constitutes the first step for most of these automated systems. Nevertheless, the segmentation in histopathological images remains a challenging task since these images typically show variances due to their complex nature and may include a large amount of noise and artifacts due to the tissue preparation procedures. In our research group, we recently developed different segmentation algorithms that rely on representing a tissue image with a set of tissue objects and using the structural pattern of these objects in segmentation. In this paper, we review these segmentation algorithms, discussing their clinical demonstrations on colon tissues.
\end{abstract}

\section{Categories and Subject Descriptors}

J.3 [Computer Applications]: Life and Medical Sciences

\section{General Terms}

Algorithms

\section{Keywords}

Histopathological image analysis, tissue image segmentation, gland segmentation, texture

\section{INTRODUCTION}

Today, cancer is one of the most important health problems especially in developed and developing countries. The likelihood of curing cancer increases with early diagnosis and selection of correct treatment plan, for which the cancer

Permission to make digital or hard copies of all or part of this work for personal or classroom use is granted without fee provided that copies are not made or distributed for profit or commercial advantage and that copies bear this notice and the full citation on the first page. To copy otherwise, to republish, to post on servers or to redistribute to lists, requires prior specific permission and/or a fee.

ISABEL'11, October 26-29, Barcelona, Spain

Copyright 2011 ACM 978-1-4503-0913-4/11/10 ...\$10.00. grade is one of the most important factors. Currently, histopathological examination is the routinely used technique for cancer diagnosis and grading. However, this technique may lead to subjectivity as it relies on the visual analysis of biopsies under a microscope. In the literature, there exist numerous computational studies that provide objective measures for automated cancer diagnosis and grading [1, 2, 3]. These studies represent a tissue image extracting its quantitative features and use these features to classify the image. In image representation, most of these studies extract their features assuming that the image is homogeneous. However, this is not always the case, and segmentation should be carried out before using these representations.

The segmentation in histopathological images can be considered in two different contexts. The first one is tissue image segmentation, in which a heterogeneous tissue image is segmented into its homogeneous regions. To this end, most of the studies conduct grid analysis that divides the image into equal sized grids, characterizes the grids extracting their features at the pixel level, and classifies them using these features $[4,5,6]$. The second group is cell/gland segmentation, in which cells or glands are located on a given tissue image. For gland segmentation, it has been proposed to classify the image pixels using their intensity and/or textural features and apply simple techniques such as thresholding and morphological operators to these classified pixels for constructing glandular regions $[7,8,9]$. All these studies use features extracted at the pixel level and do not incorporate medical background knowledge into their segmentation. Nevertheless, image segmentation is closely related with human perception and the background knowledge is generally necessary to achieve successful segmentations.

In our recent studies, we proposed to incorporate the background knowledge into tissue image segmentation $[10,11]$ and gland segmentation [12] algorithms. To this end, we represent a tissue image with a set of tissue objects and use the structural pattern of these objects in segmentation. As these patterns are defined on the tissue objects but not directly on the pixel values, they are more successful to express the background knowledge and become less vulnerable to image variations and noise that are generally observed at the pixel level. In this paper, we review our recent segmentation algorithms, discussing their clinical demonstrations on colon tissues.

The remainder of this paper is organized as follows: We first give the details of tissue representation in Section 2 . Then, we review our recent algorithms that we developed for tissue image segmentation $[10,11]$ and gland segmen- 


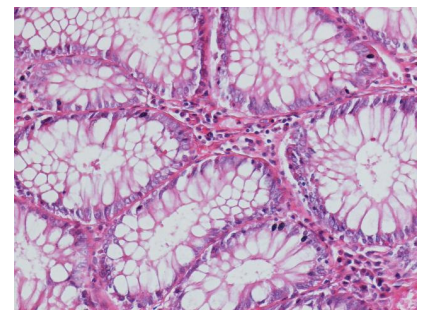

(a)

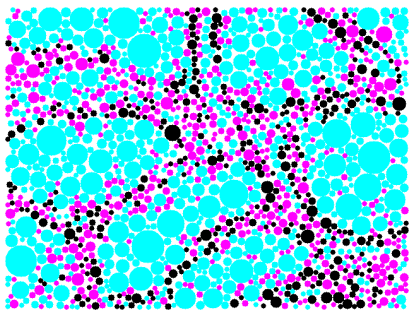

(b)
Figure 1: (a) An example of a tissue image and (b) its circular objects.

tation [12] in Sections 3 and 4, respectively. We present the experimental results obtained on colon tissue images in Section 5. Finally, we conclude the paper in Section 6 .

\section{TISSUE REPRESENTATION}

The cytological components are found in a tissue in a particular organization. This organization defines the normality and abnormality as well as the hierarchical structures in a tissue. Our segmentation algorithms rely on identifying these tissue components and modeling their structural organization. In our modeling, we approximately locate these components since the identification of their exact locations emerges a more difficult segmentation problem. To this end, we first cluster the tissue image pixels into three based on their color values using the k-means algorithm. Here we select the cluster number as three since hematoxylin-andeosin, which is the routinely used staining technique, gives three main colors: white corresponding to luminal regions and epithelial cell cytoplasms, pink corresponding to stromal regions and cell cytoplasms, and purple corresponding to cell nuclei. Then, for each cluster, we apply the morphological operators to its pixels to eliminate noise and locate a set of circular objects on its pixels using a heuristic algorithm that we proposed in [11]. These objects are herein referred to as lumen, stroma, and nucleus objects, respectively.

After this transformation, an image is represented with a set of objects instead of its pixels. Each object is characterized by its centroid, cluster type, and area. In our segmentation algorithms, we make use of these characteristics to quantify the structural organization of the tissue. An example image and its object set are shown in Figure 1; in this figure, lumen, stroma, and nucleus objects are shown with cyan, pink, and black, respectively.

\section{TISSUE IMAGE SEGMENTATION}

In this section, we review the two recent algorithms that we designed for tissue image segmentation. Both of these algorithms define their texture descriptors on the tissue objects instead of pixel values. The main difference between these algorithms is the way that they define their texture descriptors. The first algorithm [10] quantifies the object distribution using color graphs whereas the second one [11] quantifies how uniform the objects are distributed in size and space. We herein refer them to as the graphRLM and objectSEG algorithms, respectively. We provide their details in the following subsections.

\subsection{GraphRLM Algorithm}

After transforming a tissue image into the object domain, we construct a Delaunay triangulation on the objects and color the triangle edges depending on the type of their end nodes. We then define a run length matrix over the constructed graph where a run is defined as a path on which every edge has the same color. This definition is adapted from that of a gray-level run-length matrix [13] such that an edge color in our definition corresponds to a gray level in the original definition. In our algorithm, we construct the graph run-length matrix of each particular object considering the paths originated from that object. We generate the paths by running a breadth first search algorithm that starts from this particular object and visits every object in a given window. Subsequent to matrix construction, we characterize an object by accumulating the graph runlength matrices of the others that fall into a window located at the center of the object and extracting texture descriptors from the accumulated matrix. These descriptors are the short path emphasis, long path emphasis, edge type nonuniformity, and path length nonuniformity. They correspond to the gray-level run-length matrix features [13]; the feature definitions modified for the graph run-length matrices can be found in [10].

For segmentation, we employ a region growing algorithm on the constructed graph. In this algorithm, we first remove graph edges if the distance between their end objects is greater than a given distance threshold. Then, we eliminate smaller connected components and take the remaining ones as the initial seeds. We iteratively grow these initial seeds attaching the remaining objects to one of the seeds. For that, we consider all objects adjacent to at least one initial seed and attach an object to its closest seed if the distance between the object and the seed is smaller than the threshold, which is increased by its 10 percent at every iteration. After region growing, we merge the grown seeds (connected components) if their distance is smaller than a merge threshold.

\subsection{ObjectSEG algorithm}

In the objectSEG algorithm, we group the lumen, stroma, and nucleus objects into two according to their sizes so that there are a total of six different object types. To characterize a pixel, we locate a window at the center of the pixel and extract texture descriptors considering the objects that fall into that window. For each particular object type, two descriptors are defined to quantify how uniform the objects of that type are distributed in size and space; smaller values indicate more uniform regions. To measure the object size uniformity, we calculate the standard deviation of the areas of objects belonging to that particular type. To measure the object spatial distribution uniformity, we define a vector from the center point to every object of that type and compute the magnitude of the sum of these vectors.

After defining 12 descriptors for every pixel, we achieve segmentation using a region growing algorithm on them. In the first step, we select the pixels for which all descriptors are smaller than the thresholds computed on all image pixels. We consider the large connected components of these pixels as initial seeds. We then compute the descriptors for a smaller window and identify additional initial seeds using its outputs. In the second step, we iteratively grow the seeds until all pixels are assigned to a seed. For that, we 
Table 1: The quantitative results for tissue image segmentation. These results are obtained when the parameter set is selected among the ones that give the maximum of 10 regions.

\begin{tabular}{|l|l|l|l|l|l|l|l|l|}
\hline & \multicolumn{4}{|c|}{ Training set } & \multicolumn{3}{c|}{ Test set } \\
\cline { 2 - 9 } & Sensitivity & Specificity & Accuracy & Region no & Sensitivity & Specificity & Accuracy & Region no \\
\hline GraphRLM [10] & $92.8 \pm 12.4$ & $91.5 \pm 17.9$ & $93.3 \pm 7.3$ & $6.1 \pm 1.8$ & $95.6 \pm 6.5$ & $92.6 \pm 13.1$ & $94.8 \pm 4.9$ & $5.9 \pm 1.4$ \\
\hline ObjectSEG [11] & $89.0 \pm 19.9$ & $83.8 \pm 25.1$ & $87.6 \pm 11.8$ & $5.3 \pm 1.6$ & $93.1 \pm 17.4$ & $89.3 \pm 17.3$ & $92.6 \pm 9.1$ & $6.0 \pm 1.9$ \\
\hline JSEG [14] & $82.4 \pm 22.9$ & $89.7 \pm 14.3$ & $87.9 \pm 7.9$ & $6.5 \pm 2.1$ & $89.3 \pm 17.8$ & $88.6 \pm 14.9$ & $90.4 \pm 6.7$ & $7.8 \pm 2.7$ \\
\hline GBS [15] & $65.0 \pm 26.2$ & $81.5 \pm 18.7$ & $77.0 \pm 8.4$ & $6.8 \pm 1.7$ & $62.3 \pm 32.2$ & $76.3 \pm 27.5$ & $73.7 \pm 9.7$ & $4.9 \pm 1.6$ \\
\hline
\end{tabular}
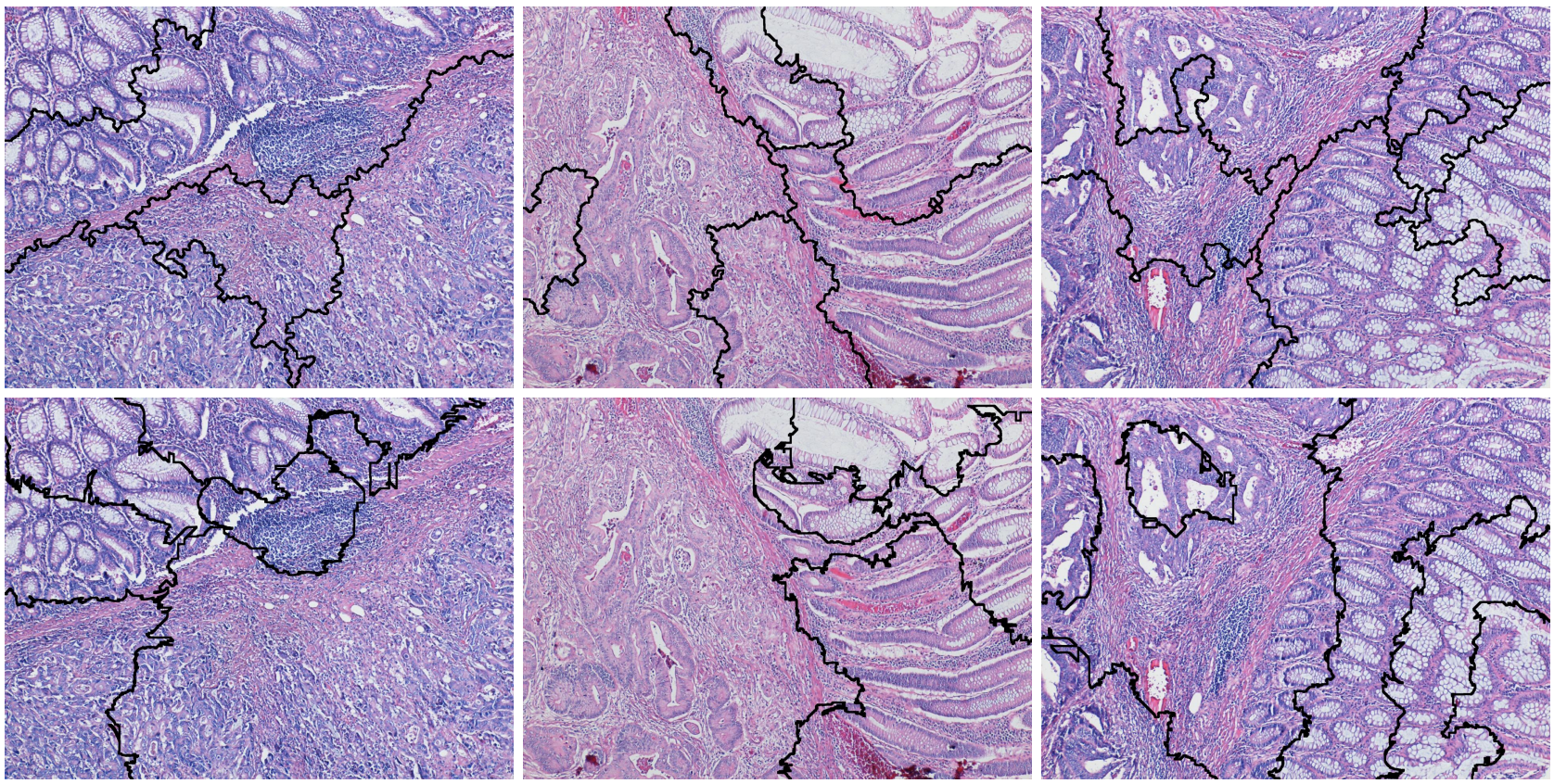

Figure 2: The visual results obtained by our tissue segmentation algorithms. These results are obtained by the graphRLM algorithm [10] (the first row), and the objectSEG algorithm [11] (the second row).

recompute the thresholds on the non-seed pixels and select those whose descriptors are all smaller than the corresponding thresholds. Likewise, we take the large connected components of the selected pixels. If a component is adjacent to a seed, we combine them. Otherwise, we consider it as a new seed. This step often gives oversegmented results, and thus, a merge step is usually necessary. In the merge step, we first merge small sized regions to their closest neighbors. We then merge two large sized regions if their distance is smaller than a merge threshold. Here we characterize each region extracting two features based on its object distribution.

\section{GLAND SEGMENTATION}

Our gland segmentation algorithm [12] relies on differentiating lumen objects into gland and non-gland objects and using the gland objects together with nucleus objects to locate the glandular structures. For this purpose, we construct an object graph around each lumen and use its local features in characterization. For a lumen object $L$, this graph includes the object itself, its $N$-closest lumen and $N$-closest nucleus objects, and the edges defined between $L$ and its closest neighbors. On this constructed graph, we define the following local features: the area of the objects, the length of the graph edges, the angles between the lumen-lumen edges, and the angles between the lumen-nucleus edges. Using these features, we cluster the lumen objects into two with the k-means algorithm. We select the cluster whose objects have a larger size on the average and consider those that belong to this cluster as the gland objects.

In order to find the inner regions of glands, we use the gland objects and another graph constructed on the nucleus objects. We construct this graph assigning an edge between each nucleus object and its $M$-closest nucleus neighbors. Then, we run a region growing algorithm on the gland objects where the pixels of the nucleus graph edges form barriers to stop the region growing. At the end of this step, we obtain candidate inner regions. Before finding their boundaries, we eliminate relatively smaller inner regions, which are typically grown from small isolated gland objects. To obtain the boundaries of each inner region, we select the nucleus objects that are closer to the inner region, form a simplified polygon on the selected objects, and dilate the polygon.

In the last step of our algorithm, we eliminate the false gland regions. To this end, we extract features from each gland candidate and learn elimination rules on these features with a decision tree classifier. The features include the area and the percentage of the pixels that were previously quantized as white, pink, and purple using the k-means al- 
Table 2: The quantitative results for gland segmentation.

\begin{tabular}{|l|l|l|l|l|l|l|}
\hline & \multicolumn{3}{|c|}{ Training set } & \multicolumn{3}{c|}{ Test set } \\
\cline { 2 - 6 } & Sensitivity & Specificity & Accuracy & Sensitivity & Specificity & Accuracy \\
\hline Object-graphs [12] & $83.4 \pm 7.7$ & $92.3 \pm 5.8$ & $88.0 \pm 4.2$ & $85.8 \pm 6.7$ & $89.1 \pm 10.4$ & $87.6 \pm 5.0$ \\
\hline Nuclei-identification [7] & $55.9 \pm 28.5$ & $55.2 \pm 32.5$ & $56.3 \pm 18.3$ & $53.8 \pm 25.7$ & $51.7 \pm 33.6$ & $53.2 \pm 13.6$ \\
\hline Lumina-identification [8] & $47.2 \pm 29.8$ & $92.7 \pm 8.4$ & $68.6 \pm 12.7$ & $52.6 \pm 32.9$ & $87.5 \pm 15.1$ & $67.6 \pm 17.2$ \\
\hline
\end{tabular}
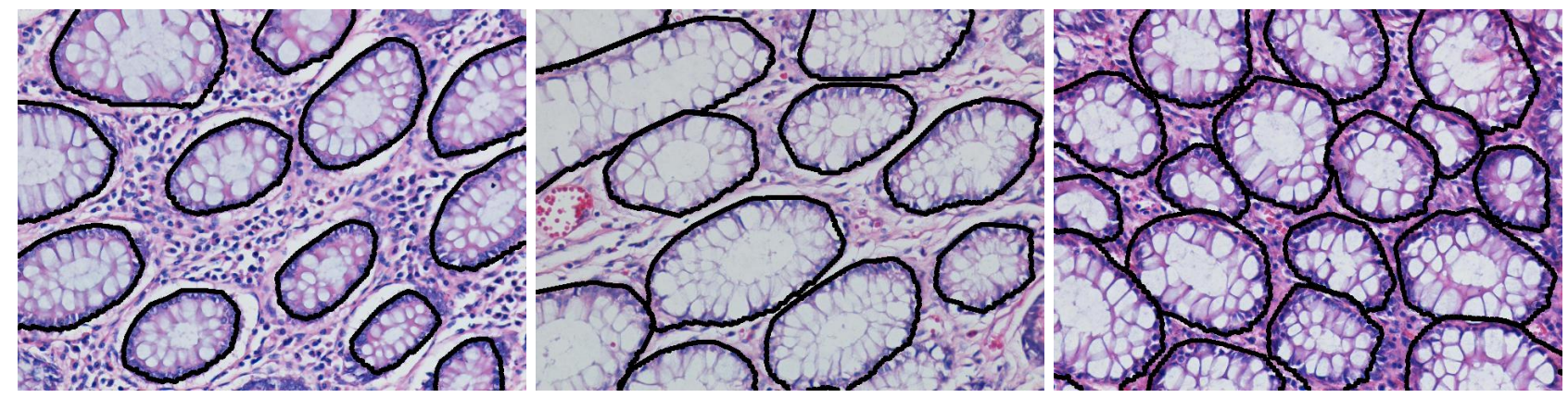

Figure 3: The visual results obtained by our gland segmentation algorithm [12].

gorithm. Here we extract these features both for the outer and inner regions of a gland candidate and use all of them in the decision tree classifier.

\section{EXPERIMENTS}

We conduct our experiments on the images of colon tissue samples. These samples are stained with the routinely used hematoxylin-and-eosin technique. Their images are taken with a Nikon Coolscope Digital Microscope. We use two different sets of images in our experiments. The first one is for tissue image segmentation experiments. This set contains 150 images that are taken using $5 \times$ microscope objective lens and $1920 \times 2560$ pixel resolution. The second set is for gland segmentation experiments. This set contains 72 images that are taken using $20 \times$ microscope objective lens and $480 \times 640$ pixel resolution.

Both of these sets are divided into training and test sets. For gland segmentation, the training set is used to learn the decision tree classifier and estimate the parameters of the algorithms that we use in our comparisons. For tissue image segmentation, the training set is used to estimate the model parameters. In estimation, we select the parameter set that gives the highest segmentation accuracy on the training samples. In tissue image segmentation experiments, we consider only the parameter sets that yield at most 10 segmented regions in order to avoid oversegmentations. The details of the parameter selection together with the candidate parameter values can be found in our previous studies $[10,12]$.

In tissue image segmentation experiments, the aim is to obtain homogeneous regions that include either normal or cancerous cells. In Figure 2, we provide the visual results of example images obtained by the graphRLM (the first row) and objectSEG (the second row) algorithms. As seen in these examples, normal and cancerous regions are successfully separated from each other, especially when the graphRLM algorithm is used. In order to obtain quantitative results, we calculate the accuracy, sensitivity, and specificity percentages, comparing the images with their gold standards. The average of these percentages and their stan- dard deviation are given in Table 1 . In this table, we also provide the quantitative results obtained by two other algorithms. These are the JSEG [14] and graph-based segmentation (GBS) [15] algorithms, which are implemented for generic images but not specifically designed for histopathological images. The quantitative results show that especially the graphRLM algorithm improves the results of these generic image segmentation algorithms, indicating the importance of designing segmentation algorithms for specific applications.

In gland segmentation experiments, the aim is to locate glandular structures in a given image. In our experiments, we focus on locating the glands in normal tissues. In Figure 3, we provide the visual results obtained for example images. The visual results show that the proposed algorithm gives successful results for finding the gland locations. In Table 2, we report the accuracy, sensitivity, and specificity percentages obtained on the training and test sets. In this table, we also provide the results obtained by two other algorithms. The first algorithm (nuclei-identification) finds epithelial cell nucleus pixels by thresholding, dilates these pixels, and fills the holes in between these dilated pixels. It then identifies the large enough components as glands [7]. The second algorithm (lumina-identification) identifies nucleus and lumen pixels by thresholding and applies the iterative region growing algorithm to obtain glands [8]. The quantitative results show that our object-graph approach improves the results, indicating the usefulness of employing object-level information in segmentation. Please note that the other algorithms can obtain relatively better results when their parameters are fine-tuned image by image. However, one parameter set that yields good results for an image usually gives worse results for the others.

\section{CONCLUSION}

In this paper, we review the segmentation algorithms that we recently developed in our research group [10, 11, 12]. These algorithms represent a tissue image with a group of objects, which approximately correspond to tissue compo- 
nents, and make use of the structural organization of these objects in segmentation. The experiments on colon tissue images show that these algorithms yield better results for tissue image segmentation and gland segmentation.

\section{ACKNOWLEDGEMENTS}

We would like to thank the Scientific and Technological Research Council of Turkey for the financial support (grant number TÜBİTAK 109E206).

\section{REFERENCES}

[1] Altunbay, D., Cigir, C., Sokmensuer, C., and Gunduz-Demir, C. 2010. Color graphs for automated cancer diagnosis and grading. IEEE Transactions on Biomedical Engineering. 57, 3, 665-674.

[2] Doyle, S., Feldman, M., Tomaszewski, J., and Madabhushi, A. 2011 (in press). A boosted Bayesian multi-resolution classifier for prostate cancer detection from digitized needle biopsies. IEEE Transactions on Biomedical Engineering.

[3] Sertel, O., Kong, J., Shimada, H., Catalyurek, U. V., Saltz, J. H., and Gurcan, M. N. 2009. Computer-aided prognosis of neuroblastoma on whole slide images: classification of stromal development. Pattern Recognition. 42, 6, 1093-1103.

[4] Smolle, J. 2000. Computer recognition of skin structures using discriminant and cluster analysis. Skin Research and Technology. 6, 2, 58-63.

[5] Mete, M., Xu, X., Fan, C.-H., and Shafirstein, G. 2007. Automatic delineation of malignancy in histopathological head and neck slides. $B M C$ Bioinformatics. 8, Suppl 7, S17.

[6] Wang, Y., Crookes, D., Eldin, O. S., Wang, S., Hamilton, P., and Diamond, J. 2009. Assisted diagnosis of cervical intraepithelial neoplasia (CIN). IEEE Journal of Selected Topics in Signal Processing. $3,1,112-121$.

[7] Wu, H.-S., Xu, R., Harpaz, N., Burstein, D., and Gil, J. 2005. Segmentation of microscopic images of small intestinal glands with directional 2-D filters. Analytical and Quantitative Cytology and Histology. 27, 5, 291-300.

[8] Wu, H.-S., Xu, R., Harpaz, N., Burstein, D., and Gil, J., 2005. Segmentation of intestinal gland images with iterative region growing. Journal of Microscopy. 220, 3, 190-204.

[9] Farjam, R., Soltanian-Zadeh, H., Jafari-Khouzani, K., and Zoroofi, R. A. 2007. An image analysis approach for automatic malignancy determination of prostate pathological images. Clinical Cytometry. 72B, 4, 227-240.

[10] Tosun, A. B. and Gunduz-Demir, C. 2011. Graph run-length matrices for histopathological image segmentation. IEEE Transactions on Medical Imaging. 30, 3, 721-732.

[11] Tosun, A. B., Kandemir, M., Sokmensuer, C., and Gunduz-Demir, C. 2009. Object-oriented texture analysis for the unsupervised segmentation of biopsy images for cancer detection. Pattern Recognition. 42, 6, 1104-1112.

[12] Gunduz-Demir, C., Kandemir, M., Tosun, A. B., and Sokmensuer, C. 2010. Automatic segmentation of colon glands using object-graphs. Medical Image Analysis. 14, 1, 1-12.

[13] Galloway, M. M. 1975. Texture analysis using gray level run lengths. Computer Graphics and Image Processing. 4, 172-179.

[14] Deng, Y. and Manjunath, B. S. 2001. Unsupervised segmentation of color-texture regions in images and video. IEEE Transactions on Pattern Analysis and Machine Intelligence. 23, 8, 800-810.

[15] Felzenszwalb, P. F. and Huttenlocher, D. P. 2004. Efficient graph-based image segmentation. International Journal of Computer Vision. 59, 2, 167-181. 